\title{
Working in partnership with probation
}

\section{The first two years of a mental health worker scheme in a probation service in Wandsworth}

\author{
Andrea Cohen, Nick Bishop and Matthew Hegarty
}

\begin{abstract}
Aims and method To describe the characteristics of probationers assessed $(n=83)$ and supported $(n=31)$ by a mental health worker (MHW) attached to a probation service. A survey of cases and case descriptions. Results Referrals were predominantly White, unemployed males on probation orders. Most had acquisitive/property and violent convictions along with substance misuse problems and personality disorder diagnoses. The MHW supported probationers already in contact with psychiatric services and provided a safety net, and sometimes sole support, to those who were not.

Clinical implications The MHW helped to bridge the gap between criminal justice and mental health systems by facilitating contact between probationers and psychiatric services. The clinical effectiveness of the scheme should now be examined.
\end{abstract}

Government policy has called for the diversion of mentally disordered offenders (MDOs) from the criminal justice system and of multi-agency working with this group (Home Office, 1990: Department of Health \& Home Office, 1992). The Inner London Probation Service responded by forming a partnership with the Mental After Care Association. Together, they appointed a mental health worker (MHW) attached to Highbury Corner Magistrate's Court (Burney, 1993). The success of this project led to the appointment of MHWs in three other inner London boroughs. The Mental After Care Association assumed responsibility for managing these schemes and funding was provided by the Inner London Probation Service, local health authorities and charitable organisations.

\section{The study}

This study examines the first 30 months of an MHW scheme in the London borough of Wandsworth, which began in May 1994. The role of the MHW evolved to fit specific local needs: (a) To assist probation officers in the supervision of MDOs.

(b) To work with probation officers to improve assessment of MDO needs through consultation and training, and contribute to pre-sentence reports and supervision plans.

(c) To liaise with other agencies regarding individual MDOs and facilitate access to services.

(d) To carry a caseload of probation clients with mental health needs, particularly those reluctant to engage with psychiatric services.

Referral criteria were purposely loose. Guidelines simply stated that suitable referrals should "have or be known to have a mental health problem and a potential unmet need for mental health intervention".

This study describes cases assessed by the MHW and those incorporated into a continuing care group (CCG). A brief composite case description is also provided.

Standardised forms were completed prospectively by the MHW, which tapped information about all assessments and CCG contacts. A descriptive analysis was carried out and a composite case description was compiled using cases seen during the study period.

\section{Findings}

\section{Assessments}

Socio-demographic details. The MHW conducted 83 assessments involving 76 individuals: an average of 33 assessments per annum. Approximately $3 \%$ of the annual probation caseload and approximately $0.5 \%$ of the annual pre-sentence report caseload were assessed by the MHW.

The majority of those assessed were male $(n=76 ; 92 \%)$ and the overall average age was 32 
years (s.d.=8.8; range $=18-54$ years). The ethnicity of most clients was described as 'White' ( $n=49 ; 59 \%)$, followed by those labelled 'Black' $(n=30 ; 36 \%)$, 'Asian' $(n=1 ; 1 \%)$ and 'other' $(n=3$; $4 \%)$. Only four cases (5\%) were employed at the time of assessment.

Source of referral. Most cases were referred by a probation officer $(n=74 ; 89 \%)$ or other court workers (e.g. court diversion scheme, duty officer) $(n=6 ; 7 \%)$. One case was referred by a community mental health team (CMHT) and two were self-referrals.

Index offences. There were 144 index offences (i.e. offences that immediately preceded referral to the MHW), the most common being property/ acquisitive ( $n=62 ; 43 \%)$, violent $(n=36 ; 25 \%)$ and summary offences (e.g. breach of court orders) $(n=12 ; 8 \%)$.

Current orders/sentences. Most cases were on probation orders $(n=31 ; 38 \%)$ and eight $(10 \%)$ were sentenced prisoners (two of the latter were in institutions for young offenders). Seven cases $(8 \%)$ were on suspended sentence supervision orders, five $(6 \%)$ were on combination orders and six (7\%) were life-licensees. A small number had conditional discharges $(n=2 ; 2 \%)$ or were bound over $(n=1 ; 1 \%)$.

Twenty-three cases (28\%) were not subject to any order or sentence at the time of assessment (they were at the pre-sentence stage or their order/sentence had expired). Seven of these were remand prisoners.

Previous convictions. A total of 202 previous convictions were recorded for 71 cases. Property/ acquisitive offences were most common ( $n=113$; $56 \%)$, followed by violent ( $n=40 ; 20 \%)$, drug $(n=15 ; 7 \%)$ and sexual offences $(n=7 ; 3 \%)$.

Psychiatric diagnoses and psychiatric treatment. Thirty-nine cases $(47 \%)$ had one previous psychiatric diagnosis and $16(19 \%)$ had multiple diagnoses (Fig. 1). Forty (48\%) were receiving psychiatric treatment at the time of assessment; $18(45 \%)$ of these were CMHT out-patients, 11 (28\%) were in-patients and six $(15 \%)$ were receiving treatment in prison. The remainder $(n=5)$ were receiving treatment from a general practitioner (GP), counsellor, the voluntary sector and a methadone-treatment programme.

History of substance misuse. Although there were 10 clients with a previous formal diagnosis involving substance misuse, 59 (71\%) had a reported history of substance misuse: alcohol misuse ( $n=17 ; 29 \%)$, drug misuse ( $n=24 ; 41 \%)$ and alcohol and drug misuse in combination $(n=18 ; 31 \%)$.

Outcome of MHW assessments. A mental health need was identified in 76 cases $(92 \%)$. Table 1 illustrates the outcome of the MHW assessments.

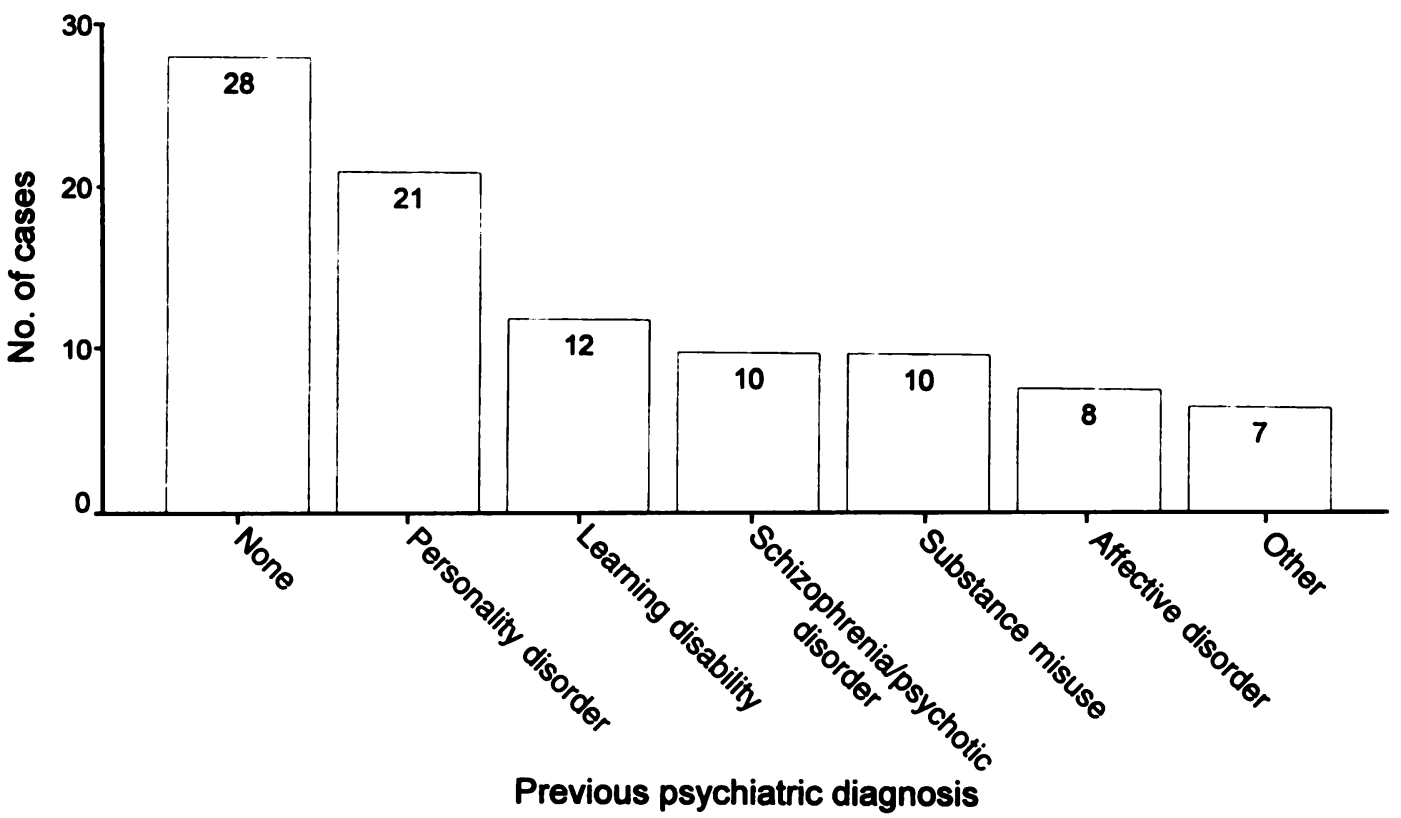

Fig. 1. Previous psychiatric diagnoses 
Table 1. Outcome of mental health worker (MHW) assessments

\begin{tabular}{ll}
\hline Outcome of MHW aseesement & $\begin{array}{l}\text { No. of } \\
\text { cases (\%) }\end{array}$ \\
\hline Admission to CCG & $31(37)$ \\
Referral to CMHT & $23(28)$ \\
Liaison with CMHT & $21(25)$ \\
Referral to forensic psychiatric service & $15(18)$ \\
Referral to mental health hostel & $13(16)$ \\
Referral to housing department & $12(14)$ \\
Referral to social services & $11(13)$ \\
Referral to drug and alcohol service & $3(4)$ \\
Referral to learning disability service & $3(4)$ \\
Registration with GP & $4(5)$ \\
Crisis intervention by MHW & $6(7)$ \\
Other & $4(5)$ \\
No mental health need identified & $7(4)$ \\
Refused intervention/impossible to engage & $11(13)$ \\
\hline
\end{tabular}

CCG, continuing care group: CMHT, community mental health team; GP, general practioner.

\section{Continuing care group (CCG)}

Characteristics. Thirty-one of the 76 individuals who were identified as having an unmet mental health need were incorporated into a CCG. Seventeen of these were in touch with psychiatric services at the time but were thought to require additional input from the MHW. Seven of these were being treated as psychiatric out-patients, five as in-patients, four by the prison medical service and one by a learning disability team. The majority of CCG members $(n=27 ; 87 \%)$ had a previous psychiatric diagnosis, most commonly personality disorder $(n=12)$, learning disability $(n=6)$, schizophrenia/psychotic disorder $(n=5)$ and affective disorder $(n=4)$. Twenty-two (71\%) had a history of substance misuse, although only three were formally diagnosed as such.

Contacts. A total of 492 MHW contacts were planned with CCG members but clients failed to attend 110 appointments (22\%). Of the 382 actual contacts, $352(92 \%)$ were face to face and $179(51 \%)$ of these were made with other professionals, most often probation officers/ assistants $(n=122)$, community psychiatric nurses/keyworkers $(n=17)$ and social workers $(n=13)$. Direct contacts took place most frequently at the local probation office $(n=116$; $33 \%)$ or at the client's home ( $n=91 ; 26 \%)$, and a wide range of other venues were also used (e.g. psychiatric units, prisons, day-centres, courts, hostels, police stations). Eighteen (58\%) CCG members were discharged over the study period.

The nature of the MHW's work is best illustrated by a 'hybrid' case example:

' $X$, a single. White 36 -year-old male, was referred by his probation officer while nearing the end of a 21 - month prison sentence for assault. ' $\mathrm{X}$ ' left school with no qualifications and had a history of long-term unemployment and homelessness. He had an extensive criminal history beginning in adolescence. with 41 previous convictions including theft, shoplifting, burglary, criminal damage and assault, and had served eight custodial sentences.

' $X$ ' had no contact with psychiatric services until just prior to his last imprisonment, when he was seen by a mental health team for the single homeless. At this time he received a diagnosis of personality disorder and alcohol misuse. He had a history of intermittent cannabis and amphetamine misuse.

The main reason for referral was so that the MHW could facilitate a link between ' $\mathrm{X}$ ' and local psychiatric services, and to assist in finding him suitable hostel accommodation after his release from prison.

Following his release from prison. ' $X$ became part of the MHW's CCG and was seen for approximately one hour per week for 18 months. The MHW initially re-linked ' $X$ ' with the mental health team for the single homeless that had treated him prior to imprisonment, and arranged a number of interim hostel placements. The MHW continued to supervise ' $\mathrm{X}$ ' on a voluntary basis after his prison license expired because his homelessness, unstable mental health. substance abuse and risk of re-offending remained unresolved. The MHW assisted ' $X$ with applications for mental health hostels, liaised with social services to secure assessment for funding and facilitated his engagement with the local CMHT. The MHW helped to facilitate smooth transfers between hostels and mental health teams, and provided supportive counselling to ' $X$ as he gained insight into his mental health problems and his need to engage with mental health services. The MHW intervened during crisis situations when ' $\mathrm{X}$ ' tried to poison himself and when he became threatening and violent following rejection by a hostel.

' $X$ ' was discharged from the CCG once he had engaged sufficiently with local psychiatric services and settled satisfactorily into his new hostel.

\section{Comment}

The probation service referred approximately $3 \%$ of its annual caseload to the MHW for assessment. It is unfortunate that probation statistics were unreliable or not directly comparable with our data, because it would have been interesting to determine how representative the MHW clients were of the probation service caseload.

It is unclear to what extent the MHW scheme affected probation service referrals to mental health (or other) services, or to what extent it ameliorated the burden of responsibilities reported by probation officers in relation to MDOs (Joseph, 1992; Roberts et al, 1995). However, it is noteworthy that probation officers referred a number of clients who were known to be involved with local mental health services. This suggests that probation officers remained sufficiently concerned about certain clients, despite psychiatric involvement. This may reflect a failure of inter-agency working between health 
and criminal justice systems, and is worthy of further investigation.

Clients assessed were predominantly White. unemployed men with high levels of substance misuse and personality disorder diagnoses and with current and previous convictions for property/acquisitive and violent offences. It is noteworthy that there was a relatively high proportion of cases with learning disabilities. The MHW identifled unmet mental health and/or social needs in $92 \%$ of cases, even though some (49\%) were already in contact with psychiatric services. The type of unmet need varied considerably, as did the interventions provided by the MHW. The MHW provided additional support to clients already involved with local mental health services and also provided a safety net for those who were not. The MHW helped to bridge the gap between the criminal justice system and the mental health system by helping clients to engage with local mental health services and other agencies. The MHW also provided the only mental health support that some clients received. The MHW filled an important gap for a small number of clients who were no longer entitled to probation service input but who still wanted support, but not from psychiatric services. The MHW also engaged with clients who complied poorly with psychiatric services or who refused psychiatric intervention.

Nearly half of those assessed were considered to have unmet needs that were sufficient enough to warrant inclusion in the CCG. These clients were seen regularly, although they were not always easy to engage, with a $22 \%$ rate of failure to attend. The role of the MHW in dealing with the CCG was diverse, and included inter-agency liaison, individual counselling, advocacy, family visits and social support (e.g. accompanying the client to court).

The MHW also assisted probation officers in assessing and supporting clients with mental health problems, and frequently saw CCG members with probation officers and other professionals. This further highlights the interagency nature of the post.

The MHW scheme provided the probation service with support in dealing with a small but heterogeneous group of MDOs with complex needs. It also served a complementary (and, at times, supplementary) role to local psychiatric services.

The scheme received further funding after its initial contract expired, and it will now be important to obtain evidence about the clinical effectiveness of the scheme, as well as its impact on psychiatric and probation services.

\section{References}

BURNEY, E. (1993) Mentally Disordered Offenders: from Court to Community. A Study in an Inner London Magistrate's Court. London: Goldsmiths College. University of London.

DEPARTMENT OF HEALTH \& HOME OFFICE (1992) Review of Health and Social Services for Mentally Disordered Offenders and Others Requiring Similar Services: Final Summary Report. CM 2088. London: HMSO.

Home OfFice (1990) Home Office Circular 66/90: Provision for Mentally Disordered Offenders. London: Home Office.

JoSEPH, P. L. (1992) Non-custodial treatment: can psychopaths be treated in the community. Criminal Behaviour and Mental Health, 2. 192-200.

Roberts, C., Hudson, B. L. \& Cullen, R. (1995) The supervision of mentally disordered offenders: the probation officers and their relationship with psychiatrists in England and Wales. Criminal Behaviour and Mental Health. 5. 75-84.

*Andrea Cohen, Research Fellow, Matthew Hegarty, Research Assistant, Section of Forensic Psychiatry, St George's Hospital Medical School, Cranmer Terrace, London SW17 ORE; and Nick Bishop, Mental Health Worker, 79 East Hill. London SW18 2QE

*Correspondence: Department of Clinical Psychology. Salomon Centre, Broomhill Rd, Tunbridge Wells, Kent TN3 OTG 